\title{
LOCALLY COMPACT FLOWS ON CONNECTED MANIFOLDS
}

\author{
KHADIJA BEN REJEB
}

\begin{abstract}
In this paper, we completely characterize locally compact flows $G$ of homeomorphisms of connected manifolds $M$ by proving that they are either circle groups or real groups. For $M=\mathbb{R}^{m}$, we prove that every recurrent element in $G$ is periodic, and we obtain a generalization of the result of Yang [Hilbert's fifth problem and related problems on transformation groups, American Mathematical Society, Providence, RI, 1976, pp. 142-146.] by proving that there is no nontrivial locally compact flow on $\mathbb{R}^{m}$ in which all elements are recurrent.
\end{abstract}

\section{INTRODUCTION AND MAIN RESULTS}

Let $M=(M, d)$ be a metric space, and let Homeo( $M)$ be the group of all homeomorphisms of $M$ equipped with the compact-open topology. In particular, Homeo $(M)$ is a Hausdorff group and every discrete subgroup of it is closed. We denote by $i d$ the identity map of $M$.

Let $G$ be a subgroup of Homeo $(M)$. An element $g \in G$ is said to be periodic if there exists an integer $n \in \mathbb{N}^{*}$ such that $g^{n}=i d$, and it is said to be recurrent if for every $\epsilon>0$, there exists an integer $n \in \mathbb{N}^{*}$ such that

$$
d\left(g^{n}(x), x\right)<\epsilon, \forall x \in M .
$$

We denote by $\operatorname{Rec}(G)$ the set of all recurrent elements of $G$, and by $P(G)$ the set of all periodic elements of $G$;

$$
\operatorname{Rec}(G)=\{g \in G \mid g \text { is recurrent }\} ; P(G)=\{g \in G \mid g \text { is periodic }\} .
$$

The group $G$ is said to be torsion free if $P(G)=\{i d\}$, and it is said to be torsion group if $P(G)=G$. The group $G$ is said to be equicontinuous if for every $x \in M$, for every $\epsilon>0$, there exists $\eta>0$ such that

$$
\forall y \in M, d(x, y)<\eta \Longrightarrow d(g(x), g(y))<\epsilon, \forall g \in G .
$$

Let $g \in \operatorname{Homeo}(M)$. We denote by $\langle g\rangle$ the subgroup generated by $g$;

$$
\langle g\rangle=\left\{g^{n} \mid n \in \mathbb{Z}\right\} .
$$

The monothetic group generated by $g$ means the closure $\overline{\langle g\rangle}$ of $\langle g\rangle$ in Homeo $(M)$. The homeomorphism $g$ is said to be regular if the subgroup $\langle g\rangle$ is equicontinuous, and it is said to be regularly almost periodic if for every $\epsilon>0$, there exists an integer $n>0$ such that

$$
d\left(g^{m n}(x), x\right)<\epsilon, \forall x \in M, \forall m \in \mathbb{Z} .
$$

Received by the editors April 19, 2020, and, in revised form, April 1, 2021, and September 5, 2021.

2020 Mathematics Subject Classification. Primary 37B05, 37B20, 57S05, 57S10.

Key words and phrases. Locally compact flow of homeomorphisms, recurrent, periodic, circle group. 
The orbit $G(x)$ of a point $x \in M$ under $G$ is defined by

$$
G(x)=\{g(x) \mid g \in G\}
$$

A flow of homeomorphisms of $M$ is a subgroup $G=\left\{h_{t} \mid t \in \mathbb{R}\right\}$ of Homeo $(M)$ satisfying the following conditions:

(1) $\phi:(t, x) \longmapsto h_{t}(x)$ is a continuous map from $\mathbb{R} \times M$ to $M$,

(2) $\phi\left(t_{1}, \phi\left(t_{2}, x\right)\right)=\phi\left(t_{1}+t_{2}, x\right), \forall t_{1}, t_{2} \in \mathbb{R}, \forall x \in M$, and

(3) $\phi(0, x)=x, \forall x \in M$.

The flow $G$ is said to be locally compact (resp. compact) if the group $G$ is locally compact (resp. compact); it is said to be periodic if there exists $r \in \mathbb{R}^{*}$ such that $h_{r}=i d$; and it is said to be recurrent if there exists a sequence $\left(t_{k}\right)_{k}$ in $\mathbb{R}_{+}$such that $t_{k} \longrightarrow+\infty$ and $h_{t_{k}} \longrightarrow i d$.

A point $x \in M$ is said to be positively recurrent (resp. negatively recurrent) under $G$ if there exists a sequence $\left(t_{k}\right)_{k}$ in $\mathbb{R}$ such that $t_{k} \longrightarrow+\infty$ (resp. $t_{k} \longrightarrow$ $-\infty)$, and $h_{t_{k}}(x) \longrightarrow x$. The point $x$ is said to be recurrent if it is either positively recurrent or negatively recurrent, and it is called periodic under $G$ if $h_{r}(x)=x$ for some real $r \neq 0$.

In [2] (see also [6]), the authors showed that if $G$ is a flow on a $2-c e l l$, then any recurrent point $x$ under $G$ is periodic under $G$. A natural question is : Is this result still true in higher dimension? A negative answer can be given by the flow $G=\left\{h_{t} \mid t \in \mathbb{R}\right\}$ defined on the product $S^{1} \times S^{1}$ of $\mathbb{R}^{4} \simeq \mathbb{C} \times \mathbb{C}$ by

$$
h_{t}\left(z_{1}, z_{2}\right)=\left(e^{i 2 \pi \alpha t} z_{1}, e^{i 2 \pi t} z_{2}\right)
$$

where $\alpha \in \mathbb{R} \backslash \mathbb{Q}$. This flow $G$ is a group of isometries of $S^{1} \times S^{1}$, and by compactness of $S^{1} \times S^{1}$, every element $h_{t}$ of $G$ is recurrent since on compact spaces regular homeomorphisms are recurrent and isometries are always regular. It follows that $G$ is recurrent, but it is not periodic. (Note here that it is easy to see that the recurrence of some element $h_{t}$ of $G(t \neq 0)$ implies the recurrence of $\left.G\right)$. Also, the point $(1,1)$ is recurrent but it is not periodic.

In this paper, we show that for every locally compact flow $G$ on a connected $m$-manifold $M(m \geq 1)$, if $G$ is recurrent then it is periodic, and recurrent points with locally compact orbits are periodic (Theorem 1.1). In all the paper, a $m$ manifold means a topological manifold of dimension $m$. Our first main result is the following theorem; where we completely characterize locally compact flows on connected $m$-manifolds for every $m \geq 1$.

Theorem 1.1. Let $G$ be a locally compact flow on a connected m-manifold $M$. Then the following hold.

(1) If $G$ is recurrent, then it is periodic and it is a circle group.

(2) $G$ is either a circle group or a real group.

(3) Every recurrent point $x$ of $M$ with locally compact orbit $G(x)$ is periodic.

Remark 1.2.

(1) Without the condition "locally compact" for the flow $G$, the results of Theorem 1.1 fail to be true; it is sufficient to consider the flow defined by $(*)$ on $\mathbb{R}^{4}$ or on the product $S^{1} \times S^{1}$ which is recurrent but not periodic. Indeed this flow is not locally compact because it is not closed. For proving this, take a sequence in the cyclic subgroup generated by the irrational rotation $h_{1}$ which converges to some periodic rotation $R \neq i d$. Then there is no real $t$ satisfying $h_{t}=R$. 
(2) One can ask this question: Let $G$ be a locally compact flow on a connected manifold $M$, then is it true that every recurrent point $x$ of $M$ is periodic?

In [12], the authors showed that every recurrent homeomorphism of the plane $\mathbb{R}^{2}$ is periodic. Is a recurrent homeomorphism of $\mathbb{R}^{3}$ periodic? it is still an open question. However, in this paper, we give partial answers. We show that a homeomorphism of $\mathbb{R}^{3}$ which is recurrent and regular is periodic. Indeed, for every integer $m \geq 1$, we show that if $G$ is a compact Lie group of homeomorphisms of $\mathbb{R}^{m}$ or a locally compact flow on $\mathbb{R}^{m}$, then every recurrent element in $G$ is periodic (Theorem 1.3 .

\section{Theorem 1.3.}

(1) Let $G$ be a compact Lie group of homeomorphisms of $\mathbb{R}^{m}$, then $\operatorname{Rec}(G)=$ $P(G)$.

(2) Let $G$ be a locally compact flow on $\mathbb{R}^{m}$, then $\operatorname{Rec}(G)=P(G)$.

(3) A recurrent homeomorphism $h$ of $\mathbb{R}^{3}$ is periodic if and only if $h$ is regular.

In all the paper, two topological groups $G$ and $T$ are isomorphic if there is a topological isomorphism between them; that is a group isomorphism which is also a homeomorphism. For a subset $E$ of a metric space $M$, we denote by $\bar{E}$ its closure and by $\partial E$ its boundary.

\section{LOCALLY COMPACT FLOWS ON CONNECTED MANIFOLDS}

In this section, we prove Theorem 1.1 .

Lemma 2.1. Let $G$ be a flow on a metric space $M$. Then the map

$$
\begin{aligned}
\alpha: \mathbb{R} & \longrightarrow G \\
t & \longmapsto h_{t}
\end{aligned}
$$

is continuous.

Proof. Let $\left(t_{k}\right)_{k}$ be a sequence in $\mathbb{R}$ such that $t_{k} \longrightarrow t$. We show that $h_{t_{k}} \longrightarrow h_{t}$. Let $K$ be any compact subset in $M$. Let $I$ be a compact neighborhood of $t$ in $\mathbb{R}$. We know that the map

$$
\begin{aligned}
& \phi: \mathbb{R} \times M \quad \longrightarrow \quad M \\
& (t, x) \longmapsto h_{t}(x)
\end{aligned}
$$

is continuous, then its restriction $\phi_{\mid I \times K}$ to the compact subset $I \times K$ is uniformly continuous. Then for every $\epsilon>0$, there exists $\eta>0$ such that for every $u, v \in I$ and for every $x, y \in K$;

$$
|u-v|<\eta, \text { and } d(x, y)<\eta \Longrightarrow d\left(h_{u}(x), h_{v}(y)\right)<\epsilon .
$$

Since $t_{k} \longrightarrow t$, then for $\eta$ there exists $k_{0}>0$ such that for every $k \geq k_{0}$, $\left|t_{k}-t\right|<\eta$, and $t_{k} \in I$, then

$$
d\left(h_{t_{k}}(x), h_{t}(x)\right)<\epsilon, \forall x \in K .
$$

Thus $d_{K}\left(h_{t_{k}}, h_{t}\right) \longrightarrow 0$ when $k \longrightarrow+\infty$. Therefore $h_{t_{k}} \longrightarrow h_{t}$ in $G$. So, $\alpha$ is continuous.

In Proposition 2.2, we describe continuous flows on connected manifolds and their orbits. 
Proposition 2.2. Let $G$ be a continuous flow on a connected manifold $M$, then the following hold:

(1) Either $G$ is recurrent or $G$ is isomorphic to $\mathbb{R}$.

(2) For every $x \in M$, either $x$ is recurrent or the orbit $G(x)$ is homeomorphic to $\mathbb{R}$.

Proof. For showing Item (1), assume that $G$ is nonrecurrent, then $G$ is nonperiodic and the map

$$
\begin{aligned}
\alpha: \mathbb{R} & \longrightarrow G \\
t & \longmapsto
\end{aligned}
$$

is bijective, it is also continuous by Lemma 2.1 For $t, t^{\prime} \in \mathbb{R}, \alpha\left(t+t^{\prime}\right)=h_{t+t^{\prime}}=$ $\alpha(t) \alpha\left(t^{\prime}\right)$, then $\alpha$ is a homomorphism. For showing that $\alpha^{-1}: G \longrightarrow \mathbb{R}$ is continuous, we will prove that if $\left(h_{t_{k}}\right)_{k}$ is a sequence in $G$ converging to some element $h_{t}$ of $G$ then $\left(t_{k}\right)$ converges to $t$. If this is not true, then $\left(t_{k}\right)$ contains a subsequence $\left(t_{\varphi(k)}\right)$ which either converges to $\infty$ or it converges to some real $r \neq t$ and $G$ is periodic. Since $G$ is assumed to be nonperiodic, then the first case holds. If $t_{\varphi(k)} \longrightarrow+\infty$, then $G$ is recurrent since $h_{\left(t_{\varphi(k)}-t\right)} \longrightarrow i d$. If $t_{\varphi(k)} \longrightarrow-\infty$, then $\left(t-t_{\varphi(k)}\right) \longrightarrow+\infty$ and $h_{\left(t_{\varphi(k)}-t\right)} \longrightarrow i d$ implies $h_{\left(t-t_{\varphi(k)}\right)} \longrightarrow i d$ since $G$ is a topological group, and so $G$ is recurrent.

Item (2) is true by the same argument of Item (1).

Lemma 2.3 ([1]). Let $G$ be a locally compact flow on a connected m-manifold $M$, then $G$ is a Lie group.

Proposition 2.4. Let $G$ be a nontrivial locally compact flow on a connected manifold $M$, then either $\left\langle h_{1}\right\rangle$ is isomorphic to $\mathbb{Z}$ or $G$ is periodic and it is a circle group.

Proof. By Weil's Lemma [9, p. 215] either $\left\langle h_{1}\right\rangle$ is isomorphic to $\mathbb{Z}$ or the closure $H=\overline{\left\langle h_{1}\right\rangle}$ is compact. Assume that $H$ is compact. Since $G$ is assumed locally compact, then it is closed and we have $G=\left\{h_{t}: t \in \mathbb{R}\right\}=\left\{h_{n+r}: n \in \mathbb{Z}, r \in\right.$ $[0,1]\}=\psi(H \times \alpha([0,1]))$ ( $\alpha$ is the map of Lemma 2.1); where $\psi$ is the map defined by

$$
\begin{aligned}
\psi: G \times G & \longrightarrow G \\
(f, g) & \longmapsto f g .
\end{aligned}
$$

By continuity of $\psi$ (since $G$ is a topological group), we deduce that $G$ is compact. It follows that every orbit $G(x)$ is compact. By [4, Proposition 1.12, p. 53 and (9.1) $3)$ p. 121], $G(x)$ is periodic and either $G(x)=\{x\}$ or $G(x)$ is homeomorphic to a circle. By Lemma $2.3 G$ is a compact connected Lie group, and so it is a torus group. Since every orbit $G(x)$ has dimension $\leq 1$, by [10, Theorem 2, p. 246] $\operatorname{dim}(G) \leq 1$. Hence $G$ is a circle group. In particular, $G$ contains a periodic element $f \neq i d$; that is $f$ satisfies $f^{n}=i d$ for some integer $n \neq 0$. Since $f=h_{t}$ for some real $t \neq 0$, then $h_{n t}=i d$; where $n t \neq 0$. Thus $G$ is periodic.

Lemma 2.5. Let $G$ be a flow on a connected manifold $M$. If $G$ is recurrent, then there exists a sequence $\left(n_{k}\right)_{k}$ in $\mathbb{N}$ and a real $r \in[0,1]$ satisfying the following conditions :

(1) $\left(h_{n_{k}+r}\right)_{k}$ is a sequence in $\overline{\left\langle h_{1}\right\rangle}$.

(2) $n_{k} \longrightarrow+\infty$ and $h_{n_{k}+r} \longrightarrow$ id when $k \longrightarrow+\infty$. 
Proof. Assume that $G$ is recurrent, then there exists a sequence $\left(h_{t_{k}}\right)_{k}$ in $G$ such that $t_{k} \longrightarrow+\infty$ and $h_{t_{k}} \longrightarrow i d$. For every $t_{k}$, there exists $n_{k} \in \mathbb{N}$ and $0 \leq r_{k}<1$ such that $h_{t_{k}}=h_{n_{k}+r_{k}}$. Since $\left(r_{k}\right) \subset[0,1]$, then by compactness of $[0,1]$ we can assume that $r_{k} \longrightarrow r \in[0,1]$. By Lemma $2.1 h_{r_{k}} \longrightarrow h_{r}$. Since $h_{t_{k}} \longrightarrow i d$, then $h_{n_{k}}=\left(h_{1}\right)^{n_{k}} \longrightarrow h_{-r}$. So, $h_{-r} \in \overline{\left\langle h_{1}\right\rangle}$ and $\left(h_{n_{k}+r}\right)_{k}$ is a sequence in $\overline{\left\langle h_{1}\right\rangle}$ converging to the identity map $i d$.

Proof of Theorem 1.1. (1) Assume that $G$ is recurrent. By Proposition 2.4, it remains to show that if $\left\langle h_{1}\right\rangle$ is isomorphic to $\mathbb{Z}$ then Item (1) is true. So, assume that $\left\langle h_{1}\right\rangle$ is isomorphic to $\mathbb{Z}$. Then $\left\langle h_{1}\right\rangle$ is a discrete subgroup of the Lie group $G$. By Lemma 2.5, there exists a sequence $\left(h_{n_{k}+r}\right)_{k}$ in $\left\langle h_{1}\right\rangle$ (since $\left\langle h_{1}\right\rangle$ is closed) such that $h_{n_{k}+r} \longrightarrow i d$; where $r \in[0,1]$. On the other hand, $\{i d\}$ is open in $\left\langle h_{1}\right\rangle$ since $\left\langle h_{1}\right\rangle$ is discrete, then there exists an integer $n_{k_{0}}$ such that $h_{n_{k_{0}}+r}=i d$ and $n_{k_{0}}+r \neq 0$. So, $G$ is periodic. In particular, $G$ is compact, and so it is a circle group (see the proof of Proposition 2.4).

(2) Follows from Proposition 2.2 and Item (1).

(3) Let $x \in M$ be a recurrent point under $G$ such that the orbit $G(x)$ is locally compact. Assume that $x$ is nonperiodic, then the stabilizer $G_{x}$ is trivial. We will show that the map

$$
\begin{aligned}
\varphi: G & \longrightarrow G(x) \\
g & \longmapsto g(x) .
\end{aligned}
$$

is a homeomorphism. Clearly $\varphi$ is a continuous bijection. So, it suffices to prove that $\varphi$ is open. Let $U$ be any open set in $G$ and let $g \in U$. Let $V$ be a compact neighborhood of $e$ in $G$ such that $V^{-1}=V$ and $g V^{2} \subset U$.

Since $G$ is second countable as a flow of homeomorphisms of a manifold, there exists a countable sequence $\left(g_{n}\right)$ in $G$ such that $G=\bigcup_{n} g_{n} V$. Since $G(x)=$ $\bigcup_{n} g_{n} V(x)$ is locally compact, by Baire's Theorem some subset $g_{n_{0}} V(x)$ and so $V(x)$ has a nonempty interior. Then for some $v \in V, V(x)$ is a neighborhood of $v(x)$. Equivalently, $v^{-1} V(x)$ is a neighborhood of $x$. Since $g v^{-1} V(x) \subset g V^{2}(x) \subset U(x)$, then $U(x)$ is a neighborhood of $g(x)$. Hence $U(x)$ is open in $G(x)$. We conclude that $G$ is homeomorphic to $G(x)$. Therefore, $x$ is recurrent implies $G$ is recurrent, and by Item (1), $G$ is periodic, so $x$ is periodic; which is a contradiction. We conclude that $x$ is periodic.

\section{ReCURREnt homeOmorphisms of $\mathbb{R}^{m}$}

In this section, "\|. $\|$ " means the Euclidean norm on $\mathbb{R}^{m}$.

In this section, we study which recurrent homeomorphisms of the Euclidean space $\mathbb{R}^{m}$ are periodic. We show that if $G$ is a compact Lie subgroup of $H$ omeo $\left(\mathbb{R}^{m}\right)$ or a locally compact flow on $\mathbb{R}^{m}$, then every recurrent element in $G$ is periodic (Propositions 3.2 and 3.3).

Lemma 3.1. Let $f$ be a homeomorphism of $\mathbb{R}^{m}$ satisfying the following conditions:

(i) The closure $\overline{\langle f\rangle}$ is a compact Lie group.

(ii) There exists a real $c>0$ such that $\|f(x)-x\|<c$, for all $x \in \mathbb{R}^{m}$.

Then $f=i d$. 
Proof. Define a map $g$ as follows:

$$
\begin{aligned}
g: B & \longrightarrow \\
x & \longmapsto \begin{cases}\varphi f \varphi^{-1}(x), & \text { if } x \in \mathrm{B}^{o} \\
x, & \text { if } x \in \partial B\end{cases}
\end{aligned}
$$

where $B$ is the closed unit-ball of $\mathbb{R}^{m}$, and $\varphi: \mathbb{R}^{m} \rightarrow \mathrm{B}^{o}$ is the homeomorphism defined by $\varphi(x)=\frac{x}{1+\|x\|}$. Clearly $g$ is continuous on $\mathrm{B}^{o}$. For showing the continuity of $g$ at $x_{0} \in \partial B$, let $\left(x_{k}\right)_{k}$ be a sequence in $\mathrm{B}^{o}$ converging to $x_{0}$. It is easy to see that for every $x, y \in \mathbb{R}^{m}$,

$$
\|\varphi(x)-\varphi(y)\| \leq\|x-y\| \frac{1+2\|y\|}{(1+\|x\|)(1+\|y\|)} .
$$

For each $k \geq 0$, if we put $u_{k}=\inf \left(\left\|\varphi^{-1}\left(x_{k}\right)\right\|,\left\|f\left(\varphi^{-1}\left(x_{k}\right)\right)\right\|\right)$, then by $(*)$ and (ii), we obtain

$$
\left\|g\left(x_{k}\right)-x_{k}\right\| \leq \frac{c\left(1+2 u_{k}\right)}{1+2 u_{k}+u_{k}^{2}}=\delta_{k} .
$$

Since $x_{k} \longrightarrow x_{0}$, then $\left\|\varphi^{-1}\left(x_{k}\right)\right\| \longrightarrow+\infty$, and so by (ii), $\left\|f\left(\varphi^{-1}\left(x_{k}\right)\right)\right\| \longrightarrow+\infty$. Hence $u_{k} \longrightarrow+\infty$ and $\delta_{k} \longrightarrow 0$, which implies that $g\left(x_{k}\right) \longrightarrow x_{0}$. Therefore, $g$ is continuous. Since $g$ is bijective, by compactness of $B, g$ is a homeomorphism.

Now, we show that $g$ is regular. Since $\overline{\langle f\rangle}$ is compact, then by Ascoli's theorem $f$ is regular. By the fact that the map $\varphi$ satisfies the property

$$
\|\varphi(u)-\varphi(v)\| \leq\|u-v\|, \forall u, v \in \mathbb{R}^{m},
$$

we deduce that $g$ is regular at each point $x \in \mathrm{B}^{o}$. Now, we show that $g$ is regular at $x_{0} \in \partial B$. Let $W_{x_{0}}=B\left(x_{0}, \lambda\right) \cap B$ be a neighborhood of $x_{0}$ in $B$; where $B\left(x_{0}, \lambda\right)$ is an open ball in $\mathbb{R}^{m}$ centered at $x_{0}$. Let $A=G\left(\overline{W_{x_{0}}}\right)$; where $G$ is the closure of the group generated by $g$. Since $g=i d$ on the boundary $\partial B$ of $B$, there exists a subset $F$ closed in $\mathrm{B}^{o}$ with boundary containing in $\partial B$ such that $A=W_{x_{0}} \cup \bar{F}$. Since $\overline{\langle f\rangle}$ is compact, then $\varphi \overline{\langle f\rangle} \varphi^{-1}$ is a compact subgroup of Homeo $\left(\mathrm{B}^{o}\right)$, and so $G(F)=\varphi \overline{\langle f\rangle} \varphi^{-1}(F)$ is a closed subset of $\mathrm{B}^{o}$ and $G(\bar{F})$ is compact in $B$. Then $V_{x_{0}}=A \backslash G(\bar{F})$ is an open neighborhood of $x_{0}$ containing in $W_{x_{0}}$ and it satisfies $g\left(V_{x_{0}}\right)=V_{x_{0}}$. Therefore, for every integer $n \in \mathbb{Z}, g^{n}\left(V_{x_{0}}\right)=V_{x_{0}} \subset W_{x_{0}}$. Hence $g$ is regular at $x_{0}$, and so $g$ is regular.

Since $B$ is compact, then by [7, Corollary 14.3.2] $g$ is almost periodic and by [7. Theorem 5.33, p. 55], there exists a sequence $\left(g_{n}\right)_{n} \subset \overline{\langle g\rangle}$ of regularly almost periodic elements $g_{n}$ of $\overline{\langle g\rangle}$ such that $g_{n} \longrightarrow g$. By [7, Theorem 5.08, p. 50] every orbit under $\overline{\left\langle g_{n}\right\rangle}$ is 0 -dimensional. For every $x \in \mathrm{B}^{o}, \overline{\left\langle g_{n}\right\rangle}(x)=\varphi \overline{\left\langle f_{n}\right\rangle} \varphi^{-1}(x)$; where $\left(f_{n}\right)_{n}$ is a sequence in $\overline{\langle f\rangle}$. Since $\overline{\langle f\rangle}$ is a compact Lie group, then by 8 , Theorem 2.3, p. 14], $\overline{\left\langle f_{n}\right\rangle}\left(\varphi^{-1}(x)\right)$ is a compact manifold. It follows that $\overline{\left\langle g_{n}\right\rangle}(x)$ is a compact 0-dimensional manifold, so it is finite. Moreover, for every $x \in \partial B, \overline{\left\langle g_{n}\right\rangle}(x)=\{x\}$. Then $g_{n}$ is pointwise periodic, and by [10, Theorem p.224], $g_{n}$ is periodic. Since $g_{n}$ coincides with the identity on the boundary $\partial B$, then $g_{n}=i d$ [11, Newman's theorem]; this is true for every integer $n$. Since $g_{n} \longrightarrow g$, then $g=i d$; equivalently, $f=i d$.

Proposition 3.2 (Recurrent homeomorphisms in compact Lie groups). Let $G$ be a compact Lie group of homeomorphisms of $\mathbb{R}^{m}$, then $\operatorname{Rec}(G)=P(G)$. 
Proof. Clearly $P(G) \subset \operatorname{Rec}(G)$. For showing the converse inclusion, let $g \in G$ such that $g$ is recurrent. Then there exists an integer $n \in \mathbb{N}^{*}$ such that

$$
\left\|g^{n}(x)-x\right\|<1, \forall x \in \mathbb{R}^{m} .
$$

Since $g^{n} \in G$, then $\overline{\left\langle g^{n}\right\rangle}$ is a compact Lie group. So, by Lemma 3.1, $g^{n}=i d$; that is $g$ is periodic. Thus, $\operatorname{Rec}(G)=P(G)$.

Proposition 3.3 (Recurrent homeomorphisms in locally compact flows).

(1) Let $G$ be a locally compact flow on $\mathbb{R}^{m}$, then $\operatorname{Rec}(G)=P(G)$.

(2) Let $f: \mathbb{R}^{m} \longrightarrow \mathbb{R}^{m}$ be a recurrent homeomorphism of $\mathbb{R}^{m}$. If $f$ is embeddable in a locally compact flow, then $f$ is periodic.

Proof. (1) Let $f \in \operatorname{Rec}(G)$. Then $\overline{\langle f\rangle}$ is a locally compact monothetic subgroup of $G$. Then, either $\langle f\rangle$ is isomorphic to $\mathbb{Z}$ or $\overline{\langle f\rangle}$ is compact.

If $\langle f\rangle$ is isomorphic to $\mathbb{Z}$, then $\langle f\rangle$ is discrete and $\{i d\}$ is open in $\langle f\rangle$. Since $f$ is recurrent, then there exists a sequence $\left(f^{n_{k}}\right)_{k}$ in $\langle f\rangle$ such that $f^{n_{k}} \longrightarrow i d$ and $n_{k} \longrightarrow+\infty$ when $k \longrightarrow+\infty$. Then, there exists $k_{0}>0$ such that for every $k \geq k_{0}$, $f^{n_{k}} \in\{i d\}$, which implies that $f^{n}=i d$ for some integer $n \in \mathbb{N}^{*}$. So, $f$ is periodic.

Now, assume that $\overline{\langle f\rangle}$ is compact. Then $\overline{\langle f\rangle}$ is a compact Lie subgroup since $G$ is a Lie group (Lemma 2.3), and by Proposition 3.2, $f$ is periodic. Hence $\operatorname{Rec}(G)=$ $P(G)$.

(2) If $f$ is embedded in a locally compact flow $G$, then $f \in \operatorname{Rec}(G)$, and by (1) $f$ is periodic.

In Corollary 3.4, we obtain two important results for flows $G$ of homeomorphisms of $\mathbb{R}^{m}$. The first one says that if all elements in $G$ are recurrent then $G$ contains no periodic element, and the second one says that if $G$ is locally compact and every element in $G$ is recurrent then $G$ must be trivial. This last result is a generalization of the result of Yang [14] saying that compact groups of homeomorphisms of manifolds in which every element is periodic are finite.

Corollary 3.4. Let $G$ be a flow on $\mathbb{R}^{m}$ such that $\operatorname{Rec}(G)=G$, then the following hold.

(1) $P(G)=\{i d\}$.

(2) If $G$ is locally compact, then $G=\{i d\}$.

Proof. (1) Assume that $P(G) \neq\{i d\}$, then there exists a periodic element $g=h_{t_{0}} \in$ $G \backslash\{i d\}$. So, $g^{q}=i d$ for some integer $q>0$, and $h_{q t_{0}}=i d$; where $r=q t_{0} \neq 0$. Then $G=\left\{h_{t} \mid 0 \leq t<r\right\}$. By Lemma 2.1, $G=\alpha([0, r])$ is compact. Then by Lemma 2.3. $G$ is a compact Lie group, and by Proposition 3.2. $\operatorname{Rec}(G)=P(G)=G$. Then, by [14, $G$ is finite. But $G$ is connected, so $G=\{i d\}$ which contradicts the assumption that $P(G) \neq\{i d\}$. Thus $P(G)=\{i d\}$.

(2) Follows from Item (1) and Proposition 3.3. (1).

Proof of Theorem 1.3. (1) Follows from Proposition 3.2.

(2) Follows from Proposition 3.3.

(3) Let $h: \mathbb{R}^{3} \longrightarrow \mathbb{R}^{3}$ be a recurrent homeomorphism which is moreover regular. Then for every orbit $O_{x}\left(x \in \mathbb{R}^{3}\right)$, the closure $\overline{O_{x}}$ is minimal, and since $h$ is recurrent, any point $x$ is almost periodic [7, Theorem 7.05]. It follows that $\overline{O_{x}}$ is compact. Then by Ascoli's theorem, $G=\overline{\langle h\rangle}$ is a compact group, and by [13], $G$ is a Lie group. So, by (1), $h$ is periodic. 


\section{REFERENCES}

[1] Khadija Ben Rejeb, Positively equicontinuous flows, Dyn. Syst. 29 (2014), no. 4, 502-516, DOI 10.1080/14689367.2014.947243. MR3265616

[2] H. Bohr, Collected Mathematical works, Vol II, Danish Math.soc, Univ of Copenhagen (1952).

[3] Khadija Ben Rejeb and Ezzeddine Salhi, Characterizations of almost periodic homeomorphisms, Topology Appl. 158 (2011), no. 16, 2094-2102, DOI 10.1016/j.topol.2011.06.008. MR.2831894

[4] J. de Vries, Elements of topological dynamics, Mathematics and its Applications, vol. 257, Kluwer Academic Publishers Group, Dordrecht, 1993, DOI 10.1007/978-94-015-8171-4. MR.1249063

[5] Andreas Dress, Newman's theorems on transformation groups, Topology 8 (1969), 203-207, DOI 10.1016/0040-9383(69)90010-X. MR238353

[6] N. E. Foland, The structure of the orbits and their limit sets in continuous flows, Pacific J. Math. 13 (1963), 563-570. MR157060

[7] Walter Helbig Gottschalk and Gustav Arnold Hedlund, Topological dynamics, American Mathematical Society Colloquium Publications, Vol. 36, American Mathematical Society, Providence, R.I., 1955. MR.0074810

[8] V. V. Gorbatsevich, A. L. Onishchik, and E. B. Vinberg, Foundations of Lie theory and Lie transformation groups, Springer-Verlag, Berlin, 1997. Translated from the Russian by A. Kozlowski; Reprint of the 1993 translation [Lie groups and Lie algebras. I, Encyclopaedia Math. Sci., 20, Springer, Berlin, 1993; MR1306737 (95f:22001)]. MR1631937

[9] Karl H. Hofmann and Sidney A. Morris, The Lie theory of connected pro-Lie groups, EMS Tracts in Mathematics, vol. 2, European Mathematical Society (EMS), Zürich, 2007. A structure theory for pro-Lie algebras, pro-Lie groups, and connected locally compact groups, DOI 10.4171/032. MR 2337107

[10] Deane Montgomery and Leo Zippin, Topological transformation groups, Interscience Publishers, New York-London, 1955. MR0073104

[11] M.H.A. Newman, A theorem on periodic transformations of spaces, Quart. J. Math, 2 (1931), $1-9$.

[12] Lex G. Oversteegen and E. D. Tymchatyn, Recurrent homeomorphisms on $\mathbf{R}^{2}$ are periodic, Proc. Amer. Math. Soc. 110 (1990), no. 4, 1083-1088, DOI 10.2307/2047760. MR.1037216

[13] John Pardon, The Hilbert-Smith conjecture for three-manifolds, J. Amer. Math. Soc. 26 (2013), no. 3, 879-899, DOI 10.1090/S0894-0347-2013-00766-3. MR.3037790

[14] C. T. Yang, Hilbert's fifth problem and related problems on transformation groups, Mathematical developments arising from Hilbert problems (Proc. Sympos. Pure Math., Northern Illinois Univ., De Kalb, Ill., 1974), Amer. Math. Soc., Providence, R. I., 1976, pp. 142-146. Proc. Sympos. Pure Math., Vol. XXVIII. MR0425999

Higher School of Sciences and Technology of Hammam Sousse, 4011 Hammam Sousse, TUNISIA

Email address: kbrjeb@yahoo.fr, khadija.benrejeb@essths.u-sousse.tn 